\title{
Fundamental limits of 'ankylography' due to dimensional deficiency
}

ARISING FROM K. S. Raines et al. Nature 463, 214-217 (2010)

Raines et al. ${ }^{1}$ propose a method, which they call 'ankylography', for three-dimensional structure determination using single-shot diffractive imaging (SSDI). But the conclusion without limitation that the three-dimensional structure of an object is "in principle encoded into a $2 \mathrm{D}$ diffraction pattern on the Ewald sphere" and may be inverted by SSDI is inadequately substantiated and conceptually misleading. Here I point out that SSDI in general suffers from a dimensional deficiency that limits the applicability of ankylography to objects that are smallsized in at least one dimension or that are approximately two-dimensional in some other way.

The rate of reliable information transfer via a spatial or temporal channel is fundamentally limited by the channel capacity, which is determined both by the number of degrees of freedom available therein and by the obtainable signal-to-noise ratio ${ }^{2}$. The resolving powers of telescopes or radio antennas and microscopes including SSDI are all limited in much the same manner. A steep (exponential) price in signal power must be paid to obtain data rates or resolutions significantly beyond those supported by the available number of degrees of freedom ${ }^{2,3}$. SSDI uses a spatial channel characterized by a linear operator $T$ that projects any real-space amplitude with support $\left[0, l^{3}\right.$ onto the Ewald sphere. It is known ${ }^{2,4}$ that there exist a pair of orthonormal bases $\left\{u_{i}\right\}_{i \geq 1}$ and $\left\{v_{i}\right\}_{i \geq 1}$, called normal modes, and also their associated modal gains $\left\{\lambda_{i}(T)\right\}_{i} \geq 1$, all non-negative and arranged in a nondecreasing order such that $T u_{i}=\sqrt{\lambda_{i}} v_{i}, \forall i \geq 1$. For any predetermined modal cutoff threshold $\varepsilon \in(0,1), N(T, \varepsilon)=\max \left\{i: \lambda_{i}(T) \geq \varepsilon\right\}$ is the number of usable normal modes, that is, the number of usable degrees of freedom. It has been rigorously proved ${ }^{5}$ that $N(T, \varepsilon) \leq 8 l^{2}+O\left(|\log \varepsilon| l \log l+\log ^{2} \varepsilon \log ^{2} l\right)$, which grows more slowly than the number of unknowns $O\left(l^{3}\right)$ in a general three-dimensional object as its size increases. The insufficiency of the number of useable degrees of freedom would persist even if $\varepsilon^{-1}$ grew exponentially as $l$ increased, as long as the exponent grew no faster than $l^{3 / 2}$ : more specifically, so long as $|\log \varepsilon|=O\left(l^{1.5-\delta}\right)$, for any fixed small $\delta>0$.

I emphasize the fundamental nature of the limitation: that singleshot diffraction does not convey sufficient information about the three-dimensional structure of an object, even if the amplitude (instead of intensity) of the diffracted field is sampled continuously and measured directly with no phase ambiguity. Given a practically obtainable signal-to-noise ratio and a measurement accuracy that together determine a threshold $\varepsilon$ of modal cutoff, any signal in a linear space spanned by normal modes of orders higher than $N(T, \varepsilon)$ is essentially lost in transmission or attenuated beyond detection. Oversampling and inversion algorithms are irrelevant in this context.

I note that there has been substantial criticism ${ }^{6-8}$ of ankylography even before its publication ${ }^{1}$. However, no agreement seems to have been reached on the fundamental problem of dimensional deficiency in ankylography, and an upper bound for the number of degrees of freedom suggested by one critic $^{6}$ has been disputed and its applicability questioned by Raines et al. ${ }^{1,7,8}$, whose response demands a mathematically rigorous proof like the one given here. The present analysis also demonstrates the need to quantify noise and a means of doing so, in order to determine a cutoff threshold based on signal-to-noise ratio, which in turn defines the number of useable degrees of freedom. Although Raines et al. ${ }^{1,7}$ discuss degrees of freedom, matrix ranks and data sufficiency, they fail to quantify the noise. To say simply that
"Poisson noise was added" to the diffraction patterns does not specify how noise addition was implemented in their numerical simulations, which are the only justification of 'ankylography' in the absence of a theoretical base. This vagueness about noise makes it difficult to interpret the reported results and to reconcile the apparent contradiction between the authors' (ref. 1) and the critic's (ref. 6) simulation results. Raines et al. ${ }^{1,8}$ cite "full rank of matrix" and other numerical results to support the claim that a curved surface of detection and oversampling yielded sufficient information for three-dimensional structure inversion, but they have not responded to the critic's numerical examples and figures showing rapid decays of singular values ${ }^{6}$.

In summary, SSDI of truly three-dimensional structures does not scale. The applicability of ankylography is limited to objects that are small-sized with respect to the wavelength in at least one dimension or have structures that are essentially two-dimensional in complexity. Such may be the case in the computer tests and preliminary experiment of ref. 1. Raines et al. ${ }^{1}$ also emphasize certain "physical constraints", many of which are actually steps of numerical procedures instead of mathematical constraints of model formulation. Incorporating more genuine physical constraints could possibly alleviate the problem of dimensional deficiency. However, that would diminish the generality and appeal of ankylography, and the same feat is arguably achievable by conventional diffractive imaging with a flat image detector.

\section{METHODS}

With $F_{y} F_{x}$ being a two-dimensional Fourier transform and $T_{z}$ being a linear operator of $z$ integration ${ }^{5}$, the upper bound on $N(T, \varepsilon)$ follows from the wellknown result ${ }^{9}$ that $N\left(F_{x}, \varepsilon\right)=N\left(F_{y}, \varepsilon\right)=2 l+O(|\log \varepsilon| \log l)$, in conjunction with inequalities $N(T, \varepsilon) \leq 2 N\left(T_{z} F_{y} F_{x}, \varepsilon / 2\right) \leq 2 N\left(F_{y} F_{x}, \varepsilon / 2\right) \leq 2 N\left(F_{y}, \varepsilon / 2\right) N\left(F_{x}, \varepsilon / 2\right)$, with the second inequality following from an operator inequality ${ }^{10}$ $F_{x}^{*} F_{y}^{*} T_{z}^{*} T_{z} F_{y} F_{x} \leq\left\|T_{z}\right\|^{2} F_{x}^{*} F_{y}^{*} F_{y} F_{x}$

\section{Haiqing $\mathrm{Wei}^{1}$ \\ ${ }^{1}$ Ambow Research Institute, Ambow Education Group, Beijing 100088, China. \\ email: davidhwei@yahoo.com}

Received 28 January 2010; accepted 21 September 2011.

1. Raines, K. S. et al. Three-dimensional structure determination from a single view. Nature 463, 214-217 (2010).

2. Gallager, R. G. Information Theory and Reliable Communication (Wiley, 1968).

3. Hansen, R. C. Fundamental limitations in antennas. Proc. IEEE 69, 170-182 (1981).

4. Miller, D. A. B. Spatial channels for communicating with waves between volumes. Opt. Lett. 23, 1645-1647 (1998).

5. Wei, H. Fundamental limits of "ankylography" due to dimensional deficiency. Preprint at http://arXiv.org/abs/1001.4594v1 (2010).

6. Thibault, P. Feasibility of $3 \mathrm{D}$ reconstructions from a single 2D diffraction measurement. Preprint at http://arXiv.org/abs/0909.1643v2 (2010).

7. Miao, J. Response to "Feasibility of $3 \mathrm{D}$ reconstruction from a single $2 \mathrm{D}$ diffraction measurement". Preprint at http://arXiv.org/abs/0909.3500v1 (2009).

8. Miao, J. \& Chen, C.-C. 2nd Response to "Feasibility of 3D reconstruction from a single 2D diffraction measurement". Preprint at http://arXiv.org/abs/ 0910.0272v1 (2009)

9. Landau, H. J. \& Widom, H. Eigenvalue distribution of time and frequency limiting. J. Math. Anal. Appl. 77, 469-481 (1980).

10. Bhatia, R. Perturbation Bounds for Matrix Eigenvalues (SIAM, 2007)

Competing financial interests: declared none.

doi:10.1038/nature10634 


\section{Non-uniqueness and instability of 'ankylography'}

ARISING FRom K. S. Raines et al. Nature 463, 214-217 (2010)

Raines et al. ${ }^{1}$ have proposed 'ankylography' as a three-dimensional tomographic modality with a single monochromatic beam ${ }^{1}$. They believe that the three-dimensional structure of an object is "in principle determined by the $2 \mathrm{D}$ spherical pattern" that "is sampled at a sufficiently fine scale on the Ewald sphere." Although they restricted their approach to unspecified "certain circumstances", they claim potential "broad applications in the physical and life sciences". Nevertheless, the imaging principles and proposed methodology of ankylography are flawed, bringing into question its applications, which was also pointed out by others, such as in ref. 2.

In the continuous domain, if a significant portion of the Fourier spectrum is unavailable, the recovery of the corresponding object is typically unstable ${ }^{3,4}$. This type of instability is well known in imaging areas such as X-ray computed tomography and magnetic resonance imaging. For example, X-ray limited-angle tomography theoretically has a unique solution but in practice it yields serious artefacts ${ }^{5,6}$. In the limiting case, ankylography represents an extreme of limitedangle tomography. With knowledge only of an Ewald sphere, twodimensional analytic extension is impossible ${ }^{5-7}$. Given a sufficiently complex structure in the object support, its Fourier spectrum outside the surface of the Ewald sphere can uniquely and stably determine that object, and the solution from data on the surface of the Ewald sphere or another zero-measure surface is generally non-unique and highly unstable.

Let $\Omega \in \mathbb{R}^{3}$ be an open bounded set. Suppose that $u(\mathbf{r}) \in C_{0}^{4}(\Omega)$ is a compactly supported function whose Fourier transform is $\hat{u}(\xi)$, with $\mathbf{r}=(x, y, z)$ and $\xi=\left(\xi_{1}, \xi_{2}, \xi_{3}\right)$. Denoting

$g(\mathbf{r})=\left(\frac{\partial^{4}}{\partial x^{4}}+\frac{\partial^{4}}{\partial y^{4}}+\frac{\partial^{4}}{\partial z^{4}}+2 \frac{\partial^{4}}{\partial x^{2} y^{2}}+2 \frac{\partial^{4}}{\partial x^{2} z^{2}}+2 \frac{\partial^{4}}{\partial y^{2} z^{2}}+4 R^{2} \frac{\partial^{2}}{\partial x^{2}}\right) u(\mathbf{r})(1)$

we have

$$
\begin{aligned}
\hat{g}(\xi) & =\left[\left(i \xi_{1}\right)^{4}+\left(i \xi_{2}\right)^{4}+\left(i \xi_{3}\right)^{4}+2\left(i \xi_{1}\right)^{2}\left(i \xi_{2}\right)^{2}+2\left(i \xi_{1}\right)^{2}\left(i \xi_{3}\right)^{2}\right. \\
& \left.+2\left(i \xi_{2}\right)^{2}\left(i \xi_{3}\right)^{2}+4 R^{2}\left(i \xi_{1}\right)^{2}\right] \hat{u}(\xi) \\
& =\left(\xi_{1}^{4}+\xi_{2}^{4}+\xi_{3}^{4}+2 \xi_{1}^{2} \xi_{2}^{2}+2 \xi_{1}^{2} \xi_{3}^{2}+2 \xi_{2}^{2} \xi_{3}^{2}-4 R^{2} \xi_{1}^{2}\right) \hat{u}(\xi) \\
& =\left[\left(\xi_{1}^{2}+\xi_{2}^{2}+\xi_{3}^{2}\right)^{2}-4 R^{2} \xi_{1}^{2}\right] \hat{u}(\xi) \\
& =\left(\xi_{1}^{2}+\xi_{2}^{2}+\xi_{3}^{2}-2 R \xi_{1}\right)\left(\xi_{1}^{2}+\xi_{2}^{2}+\xi_{3}^{2}+2 R \xi_{1}\right) \hat{u}(\xi) \\
& =\left[\left(\xi_{1}-R\right)^{2}+\xi_{2}^{2}+\xi_{3}^{2}-R^{2}\right]\left[\left(\xi_{1}+R\right)^{2}+\xi_{2}^{2}+\xi_{3}^{2}-R^{2}\right] \hat{u}(\xi)
\end{aligned}
$$

Equation (2) shows that the Fourier transform of $g(\mathbf{r})$ is zero on the Ewald spheres centred at $( \pm R, 0,0)$ with radius $R$. From equation (2), we can construct many counterexamples to prove the non-uniqueness of ankylography.

In the discrete domain, instability of ankylography inherited from the continuous domain will be overwhelming when the number of unknowns is substantial. Ankylography incorporates non-negativity, uniformity and continuity in the hybrid-input-output framework ${ }^{8}$. It allows reconstruction of objects with a small number of degrees of freedom, but fails in our experiments even using an enhanced hybridinput-output algorithm with total variation minimization ${ }^{9,10}$ when an object matrix is not too small. Specifically, we used a $64 \times 64 \times 64$ Shepp-Logan phantom. The oversampling condition ${ }^{1}$ was satisfied. When all the Fourier modulus information was available, an accurate image was reconstructed. However, the reconstruction quality degraded rapidly as the known portion of the Fourier modulus was reduced, and became useless from a single view even with an excellent initial image (Fig. 1a). J. Miao of ref. 1 has confirmed that the current ankylography algorithm does not perform well if an object is symmetric or if the image size is much larger than $15 \times 15 \times 15$. We believe that these limitations are fundamental, and should have been
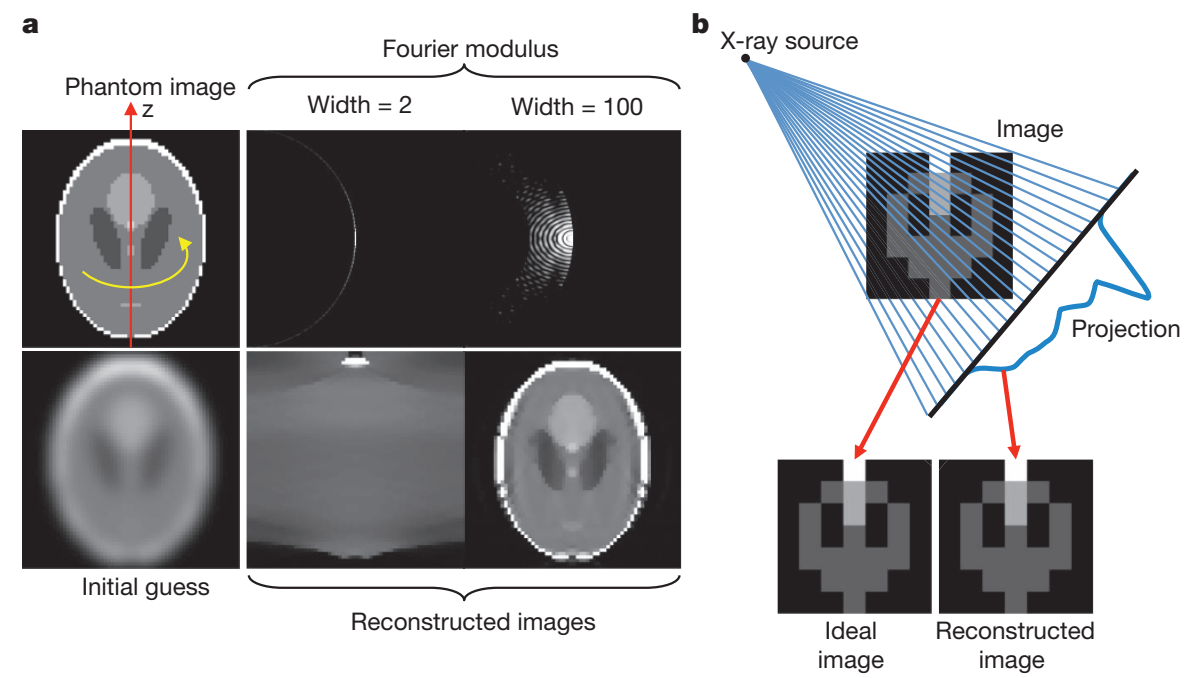

Figure 1 Numerical studies of 'ankylography' and 'one-view divergent tomography'. a, Failure of ankylography with a $64 \times 64 \times 64$ Shepp-Logan phantom (symmetric with respect to the $z$ axis) from noise-free data after 4,000 iterations. We note that the single-view measurement corresponds to the case of width $=1$ but here the case of width $=2$ is shown for better visibility of data.
The case of width $=100$ can be physically studied using an energy-sensitive detector array. $\mathbf{b}$, Success of one-view divergent tomography with a $7 \times 7$ Shepp-Logan phantom in fan-beam geometry. In this specific setting, the system matrix has a rank (the number of independent measurements) larger than the number of pixels and a reasonable condition number. 
mentioned in ref. 1. When the measured Ewald spheres form a layer of substantial thickness ( reconstruction becomes satisfactory. Data for this type of threedimensional reconstruction can be simultaneously acquired using an energy-sensitive detector array such as one from the Medipix detector family being developed by $\mathrm{CERN}^{11}$. This approach has an ultrafast imaging speed, and can be referred to as spectrography instead of extended ankylography, because the latter is characterized by its two-dimensional spherical pattern.

The methodological flaw in ankylography may also be illustrated with the example of 'one-view divergent tomography' for reconstruction of a two- or three-dimensional structure from a single fan-beam $\mathrm{X}$-ray projection ${ }^{12}$ :

$$
p(\mathbf{s}, \boldsymbol{\theta})=\int_{0}^{\infty} f(\mathbf{s}+t \boldsymbol{\theta}) \mathrm{d} t
$$

where $\mathbf{s}$ is the X-ray source position, and $\boldsymbol{\theta}$ is the unit directional vector. Following the same logic as in ref. 1, we could claim that one-view divergent tomography can reconstruct a two- or threedimensional object using the oversampling strategy. Indeed, it works well for small-size matrices (Fig. 1b). However, it is well known that the solution to this problem is non-unique and unstable, with little utility in the computed tomography field so far.

Ge Wang $^{1}$, Hengyong $\mathbf{Y u}^{1}$, Wenxiang Cong $^{1}$ \& Alexander Katsevich ${ }^{2}$ ${ }^{1}$ SBES Biomedical Imaging Division, VT-WFU School of Biomedical Engineering and Sciences, Virginia Tech, Blacksburg, Virginia 24060, USA.

email: wangg@vt.edu
${ }^{2}$ Department of Mathematics, University of Central Florida, Orlando, Florida 32816, USA.

Received 26 February 2010; accepted 21 September 2011.

1. Raines, K. S. et al. Three-dimensional structure determination from a single view. Nature 463, 214-217 (2010).

2. Thibault, P. Feasibility of $3 \mathrm{D}$ reconstruction from a single $2 \mathrm{D}$ diffraction measurement. Preprint at http://arXiv.org/abs/0909.1643v1 (2009).

3. Crimmins, T. R. \& Fienup, J. R. Uniqueness of phase retrieval for functions with sufficiently disconnected support. J. Opt. Soc. Am. 73, 218-221 (1983).

4. Foley, J. T. \& Butts, R. R. Uniqueness of phase retrieval from intensity measurements. J. Opt. Soc. Am. 71, 1008-1014 (1981).

5. Hamaker, C. etal. The divergent beam x-ray transform. Rocky Mountain J. Math. 10, 253-284 (1980).

6. Ye, Y., Yu, H. \& Wang, G. Interior tomography: mathematical analysis. In Biomedical Mathematics: Promising Directions in Imaging, Therapy Planning, and Inverse Problems (eds Censor, Y., Jiang, M. \& Wang, G.) 543-561 (Medical Physics Publishing, 2010)

7. Inouye, T. Image reconstruction with limited angle projection data. IEEE Trans. Nucl. Sci. 26, 2665-2669 (1979).

8. Fienup, J. R. Reconstruction of an object from modulus of its Fourier transform. Opt. Lett. 3, 27-29 (1978).

9. Donoho, D. L. Compressed sensing. IEEE Trans. Inf. Theory 52, 1289-1306 (2006).

10. Yu, H. \& Wang, G. Compressed sensing based interior tomography. Phys. Med. Biol. 54, 2791-2805 (2009).

11. Anderson, N. G. et al. Spectroscopic (multi-energy) CT distinguishes iodine and barium contrast material in MICE. Eur. Radiol. 20, 2126-2134 (2010).

12. Natterer, F. The Mathematics of Computerized Tomography Chs II, IV and VI (John Wiley \& Sons, 1986).

Author Contributions G.W. conceptualized and wrote the manuscript. G.W., H.Y. and W.C. identified non-uniqueness of ankylography in simple cases, and A.K. constructed the general argument. H.Y., W.C. and G.W. analysed stability of ankylography and simulated spectrography.

Competing financial interests: declared none.

doi:10.1038/nature10635 